\title{
Implantação de pacote de medidas para prevenção de infecções associadas ao cateter venoso central em crianças: percepção da equipe de enfermagem
}

\author{
Implementation of a bundle for prevention of central venous cathedral associated infections in \\ children: perception of nursing team
}

Implantación de paquete de medidas para prevención de infecciones asociadas al cateten venoso central en niños: percepción del equipo de enfermería

Gustavo Marino Ferreira Sorgi ${ }^{1}$, Natália Shinkai Binotto2*, Patrícia Basso Squarça Mendes ${ }^{3}$, Mauren Teresa Grubisich Mendes Tacla4.

\section{RESUMO}

Objetivo: Identificar a percepção da equipe de enfermagem quanto à sua participação na implantação de um pacote de medidas para prevenção de infecções associadas ao cateter venoso central em crianças. Métodos: Pesquisa exploratória, descritiva, de abordagem qualitativa, a partir de entrevistas semiestruturadas com a equipe de enfermagem das Unidade de Terapia Intensiva Pediátrica e Unidade Pediátrica de um hospital do norte do Paraná. Resultados: Após análise dos dados, emergiram três categorias: importância da implantação do pacote de medidas; participação no processo de implementação do pacote de medidas; e sugestões para melhoria do processo de implantação do pacote de medidas. Os participantes reconhecem a importância da participação na elaboração e implantação do pacote de medidas em pediatria, assim como na manutenção das práticas fundamentadas em evidências cientificas. Conclusões: Além de permitir a sistematização do cuidado, o pacote de medidas garante melhor qualidade assistencial e segurança ao paciente e profissional.

Palavras-Chave: Criança, Enfermagem Pediátrica, Equipe de enfermagem, Infecções Relacionadas a Cateter, Pacotes de assistência ao Paciente.

\section{ABSTRACT}

Objective: To identify the perception of the nursing team regarding their participation in the implementation of a bundle to prevent infections associated with the central venous catheter in children. Methods: Exploratory, descriptive, qualitative approach, based on semi-structured interviews with the nursing team of the Pediatric Intensive Care Unit and Pediatric Unit of a hospital in the north of Paraná. Results: After analyzing the data, three categories emerged: importance of implementing the bundle; participation in the process of implementing the bundle; and suggestions for improving the process of implementing the bundle. Participants recognize the importance of participating in the development and implementation of the bundle in pediatrics, as well as in the maintenance of practices based on scientific evidence. Conclusions: In addition to allowing the systematization of care, the bundle ensures better care quality and patient and professional safety.

Keywords: Child, Pediatric Nursing, Nursing team, Catheter-Related Infections, Patient Care Bundles.

\footnotetext{
${ }^{1}$ Enfermeiro. Especialista em Saúde da Criança, Universidade Estadual de Londrina (UEL), Paraná, Brasil. Tutor de Enfermagem na Universidade Norte do Paraná.

${ }^{2}$ Enfermeira. Especialista em Saúde da Criança e do Adolescente, Universidade de São Paulo (USP). Mestranda em Enfermagem, Universidade Estadual de Londrina (UEL), Paraná, Brasil. *E-mail: natalia binotto@hotmail.com

${ }^{3}$ Enfermeira. Mestre em enfermagem. Supervisora de Enfermagem da Divisão Materno-Infantil do Hospital Universitário da Universidade Estadual de Londrina (UEL), Londrina, Paraná, Brasil.

${ }^{4}$ Enfermeira. Doutora. Professora Associada do Departamento de Enfermagem, Área da Saúde da Criança e do Adolescente, Centro de Ciências da Saúde. Universidade Estadual de Londrina (UEL), Londrina, Paraná, Brasil.
} 


\section{RESUMEN}

Objetivo: Identificar la percepción del equipo de enfermería en cuanto a su participación en la implantación de un paquete de medidas para prevención de infecciones asociadas al catéter venoso central en niños. Métodos: Investigación exploratoria, descriptiva, de abordaje cualitativo, a partir de entrevistas semiestructuradas con el equipo de enfermería de las Unidad de Terapia Intensiva Pediátrica y Unidad Pediátrica de un hospital del norte de Paraná. Resultados: Después del análisis de los datos, surgieron tres categorías: importancia de la implantación del paquete de medidas; participación en el proceso de implantación del paquete de medidas; y sugerencias para mejorar el proceso de implementación del paquete de medidas. Los participantes reconocen la importancia de la participación en la elaboración e implantación del paquete de medidas en pediatría, así como en el mantenimiento de las prácticas fundamentadas en evidencias científicas. Conclusiones: Además de permitir la sistematización del cuidado, el paquete de medidas garantiza mejor calidad asistencial y seguridad al paciente y profesional.

Palabras Clabe: Niño, Enfermería Pediátrica, Grupo de Enfermería, Infecciones Relacionadas con Catéteres, Paquetes de Atención al Paciente.

\section{INTRODUÇÃO}

Em meados de 2005, o primeiro conceito de pacote de medidas (bundle, em inglês) foi criado pelo Institute for Healthcare Improvement (IHI), uma organização americana sem fins lucrativos, que visa a melhoria dos cuidados em saúde no mundo. Trata-se de um conjunto de práticas simples e já existentes, que devem ser implementadas juntas possibilitando uma ação completa na prevenção e promoção da saúde (IHI, 2011).

Apesar das práticas assistenciais recomendadas já ocorrem, ainda, encontram-se desorganizadas em meio ao processo e são executadas de maneira não uniforme pelos profissionais (IHI, 2011). Ainda, essas práticas pertencentes aos pacotes de medidas são norteadas pela Prática Baseada em Evidências (PBE) originada na década de 1970, com a intenção de solucionar lacunas na assistência à saúde por meio da aplicação de ações baseadas nas melhores evidências científicas (LACERDA et al., 2011).

A elaboração e implantação de um pacote de medidas representa uma iniciativa relevante na promoção da melhoria da qualidade assistencial e segurança ao paciente, uma vez que estudos destacam a redução das taxas de infecção hospitalares que podem predispor a inúmeras complicações de saúde (DALLÉ et al., 2012, FORTUNATTI, 2017).

Segundo a Agência Nacional de Vigilância Sanitária (ANVISA), o uso de pacotes de medidas pode resultar na redução das taxas de Infecções Primárias de Correntes Sanguíneas (IPCS) associadas ao cateter venoso central (CVC) nos cenários hospitalares, porém ressalta-se a importância da implementação das intervenções em conjunto (BRACHINE et al., 2012; BRASIL, 2017, FORTUNATTI, 2017).

Diferente do cateter venoso periférico (CVP) que representa um acesso mais rápido, menos invasivo e complexo (NOBRE; MARTINS, 2018), o CVC é indicado para quadros de gravidade e internações prolongadas, infusões de soluções e fármacos, reposição volêmica até transfusões e coleta de sangue. Além de ser amplamente utilizado em criança, a responsabilidade de manipulação e manutenção desse dispositivo venoso pela equipe de enfermagem deve compreender a importância da prevenir infecções e complicações decorrentes do seu manejo inadequado, além do impacto nos progressivos custos e tempo de hospitalização (GOMES, NASCIMENTO, 2013).

Em relação ao impacto de intervenções interdisciplinares nos indicadores de IPCS relacionada ao CVC, ressalta-se a importância do envolvimento da equipe de saúde e adesão às mudanças propostas a partir de evidências científicas, resultando no impacto positivo nos desfechos assistenciais, tanto no período precoce, quanto tardio do diagnóstico e tratamento (GOMES, NASCIMENTO, 2013, FORTUNATTI, 2017). 
Sabe-se que aplicar protocolos na prática assistencial não é uma tarefa fácil, constituindo-se um grande desafio, uma vez que os mesmos devem ser dinâmicos e construídos em conjunto com a equipe de saúde, para que todos se sintam motivados, permitindo assim, a eficácia das medidas implantadas e que o processo da assistência seja significativo e avaliado (SILVA et al., 2012).

Assim, este estudo teve como objetivo identificar a percepção dos profissionais de enfermagem quanto à participação na implantação de um pacote de medidas para prevenção das infeções associadas e relacionadas ao CVC em crianças.

\section{MÉTODOS}

Trata-se de um estudo exploratório, descritivo, de abordagem qualitativa, que deriva do projeto "Impacto da implantação de bundle de infecções associadas e relacionadas a cateter venoso central na criança", aprovado pelo Comitê de Ética em Pesquisa Envolvendo Seres Humanos da Universidade Estadual de Londrina (CEP/UEL) conforme parecer ํㅡㄴ 205/2013, CAAE: 22737413.

Participaram deste estudo dez profissionais da equipe de enfermagem, entre auxiliares, técnicos em enfermagem e enfermeiros, que atuam no cuidado direto às crianças e participaram da implantação do pacote de medidas de prevenção de infecções associadas ao CVC nas Unidade de Terapia Intensiva Pediátrica (UTIP) e Unidade Pediátrica (UP) de um hospital universitário do norte do Paraná, de nível terciário, com 20 leitos na UP e 5 na UTIP que atendem crianças de zero até 12 anos, entre várias especialidades médicas. E como critérios de exclusão, o afastamento por atestado ou férias e recusa em participar da pesquisa.

Os dados foram coletados no segundo semestre de 2014, após a obtenção da assinatura do Termo de Consentimento Livre e Esclarecido (TCLE) (BRASIL, 2012) dos participantes do estudo, a partir de entrevistas semiestruturadas com as seguintes perguntas norteadoras: "Para você, como foi a implantação do pacote de medidas para prevenção das infecções relacionadas ou associadas ao CVC na criança? Como você avalia sua participação neste processo? Quais suas sugestões para melhorar o processo?"

Ressalta-se que foram realizados dois testes-piloto para a adequação do instrumento, e posteriormente, as entrevistas foram realizadas nas UTIP e UP, gravadas e transcritas na íntegra. Os dados obtidos foram analisados com base no Método de Interpretação dos Sentidos, desenvolvido em três etapas: a leitura compreensiva do material selecionado, exploração do mesmo e a elaboração da síntese interpretativa significativo (MINAYO et al., 2012).

Na primeira etapa, foi realizada a leitura das entrevistas com a valorização das particularidades das falas dos profissionais e selecionados trechos dos depoimentos com o objetivo de identificar ideias explícitas e implícitas sobre o tema (MINAYO et al., 2012). Em seguida, surgiram questionamentos para problematizar e sugerir eixos orientadores para a interpretação dos dados: há divergências das ideias dos participantes? Há pontos convergentes entre elas?

Na terceira etapa, foi realizado um diálogo entre a fundamentação teórica, informações de outros estudos, depoimentos e seus contextos, observações em campo, objetivo da pesquisa e ideias presentes nos depoimentos (MINAYO et al., 2012).

Para preservação do anonimato dos profissionais, foram identificados com a letra E, que significa "enfermagem", seguida de um número que indica a ordem de realização das entrevistas.

\section{RESULTADOS E DISCUSSÃO}

Após análise das entrevistas com os profissionais da equipe de enfermagem, emergiram três categorias analíticas: importância da implantação do pacote de medidas, participação no processo de implantação do pacote de medidas, e sugestões para a melhoria no processo de implantação do pacote de medidas. 


\section{Importância da implantação do pacote de medidas}

O pacote de medidas de prevenção de infecções associadas ao CVC em crianças foi construído coletivamente com a equipe de enfermagem e organizado a partir dos seguintes itens: higiene das mãos antes e após a manipulação do CVC e preparo de medicações; desinfecções dos conectores e equipos com álcool por 30 segundos antes de inserir medicamentos, soro ou drogas; manter o sistema fechado; não molhar ou imergir o cateter durante o banho; formação de um time pediátrico para inserção periférica; e a criação do lema ESCEVICE (estabilização, cobertura estéril, visualização e segurança) (SQUARÇA, 2015).

Sendo assim, a importância da implantação desse pacote de medidas na prevenção da infecção do CVC, na percepção da equipe de enfermagem pode ser observado a seguir: (E5)

[...] foi muito bom eles terem implantado, porque diminui bastante a infecção dos cateteres [...]

[...] é uma medida que visa melhorar a criança. E a infecção por cateter é uma coisa perigosa, então a gente vai cuidar direito [...] (E1)

O uso do pacote de medidas está ligado diretamente à segurança do paciente, além de ser ressaltado por especialistas e organismos internacionais como método eficaz para prevenir e reduzir infecção de corrente sanguínea (BRACHINE et al., 2012).

A redução das infecções relacionadas ao acesso vascular é dependente da utilização das medidas preventivas, sendo de suma importância que os profissionais envolvidos sejam conscientizados quanto aos riscos ligados aos procedimentos, ao investimento de qualificação e a padronização de condutas, indispensáveis para uma prática segura e ética (OLIVEIRA et al., 2014; ROSADO et al., 2011).

Ainda, estudos afirmam que a implementação do pacote de medidas de prevenção de IPCS pelo uso do CVC representa uma medida complexa e relevante, que por meio da uniformização e sistematização do cuidado baseado em evidências promove melhorias nos processos assistenciais quanto à qualidade e segurança do paciente (DALLÉ et al., 2012, ROSADO et al., 2011).

[...] só a pessoa que punciona sabe como é difícil e se a criança tem um acesso bom, a gente vai ajudar a manter da melhor maneira possível, pois é melhor para criança e para nós [...] (E1)

[...] eu procuro sempre passar álcool para desconectar, cuidar bem do curativo, evitar molhar durante o banho e protegê-lo, para tudo ocorrer certo e não ter nenhum problema para criança [...] (E2)

[...] Toda a vez que eu estou com um paciente que tem um cateter eu procuro atender todas as medidas para prevenir infecção. Quanto a durabilidade do cateter, diminui também o trauma da criança, porque para acesso periférico tem que furar várias vezes, às vezes você não consegue e acaba até atrasando a medicação [...] (E3)

Observa-se que as práticas para o manejo adequado do CVC podem tornar o trabalho profissional mais célere, uma vez que esse acesso vascular possui durabilidade maior, sem a necessidade de punção periférica com frequência (GOMES et al, 2013). Entretanto, a permanência do dispositivo depende das ações profissionais para evitar a perda antes do fim da indicação, principalmente quando a remoção é acidental ou por motivo de obstruções (OLIVEIRA et al., 2014).

Ainda, o comprometimento profissional na implantação e manutenção do pacote de medidas, torna-se significativo quando as medidas de prevenção de infecções associadas ao CVC resultam diretamente na segurança e qualidade no cuidado das crianças (DALLÉ et al., 2012).

Participação no processo de implantação do pacote de medidas 
Em relação à participação no processo de implantação do pacote de medidas para prevenção de infecções associadas ao CVC, os profissionais apresentaram adesão às práticas fundamentadas:

[...] eu acho que estou fazendo a minha parte, o que eu tenho que fazer, de acordo com o que foi dado à minha função [...] (E4)

[...] eu tenho uma boa participação, porque eu faço tudo como foi orientado [...] (E5)

Além de reconhecerem a importância da participação ativa no processo, os profissionais exprimem que a aplicação das técnicas corretas na prática ocorre após compreensão significativa dos impactos na qualidade da assistência ou risco de infecções e complicações de saúde das crianças.

Nesse sentido, esforços devem ser realizados na formação profissional e na educação permanente, tendo foco na consciência crítica, conhecimento e posicionamento ético, incluindo a elaboração e seguimento dos protocolos de manuseio de acesso vascular, para a transformação da realidade ser concretizada, garantindo uma prática segura (OLIVEIRA et al., 2014; ROSADO et al., 2011).

[...] sim, porque quando você sabe, entende, acaba fazendo. [...] (E3)

[...] eu procuro fazer tudo que ela passou para gente, para tudo correr certo e não ter nenhum problema para a criança [...] (E10)

[...] a gente sabe que não pode molhar, mas não é por capricho. É conforme o protocolo, por conta disso, disso e disso. É fundamentado [...] (E9)

Estudos defendem o quanto maior for a efetividade da abordagem educacional, interdisciplinar e permanente estabelecida nos serviços de saúde, associada à elaboração de normas para inserção e manutenção de CVC, menores serão as taxas de IPCS (FORTUNATTI, 2017; ROSADO et al., 2011).

[...] relacionado ao cateter: às vezes, eu mesmo fazia por não saber e com essas aulas eu aprendi muito. [...] (E7)

[...] achava que não era para molhar no banho, porque era preguiça de trocar o curativo, então agora o funcionário já está com a visão diferente [...] (E9)

Estas falas são corroboradas por Dallé et al. (2012) que defendem a amplitude dos conhecimentos profissionais acerca das ações preventivas após participação em aprimoramentos associada à maior qualificação e motivação da equipe. Dessa forma, os profissionais capacitados, tornam-se mais motivados e passam a realizar os procedimentos de forma correta, pois adquirem conhecimentos baseados em evidências científicas (DALLÉ et al., 2012; OLIVEIRA et al, 2016).

\section{Sugestões para melhoria do processo de implantação do pacote de medidas}

Nesta categoria, surgiram divergências nas sugestões para a melhoria do processo de implantação do pacote de medidas dadas pelos participantes, portanto, originaram-se subcategorias para uma discussão mais detalhada sobre $o$ assunto.

\section{Risco de esquecimento}

[...] não deixar cair no esquecimento. Porque implantou há um mês está esquecendo? Tem que sempre estar treinando novamente, lembrando, colocando cartaz, falando para a gente como que está [...] (E2)

[...] é o que eu já falei, acho que tem que ser sempre, não pode fazer e esquecer, e tem que ser continuado [...] (E7) 
Cuidados de saúde com qualidade na assistência e focados na prevenção de erros são primordiais para minimizar os fatores de risco associados à infecção, porém há necessidade de garantir a motivação e capacitação dos profissionais, com o intuito de garantir a aplicação das práticas recomendadas no pacote de medidas (OLIVEIRA et al., 2016).

[...] é continuar dessa forma, cuidando bem do cateter, para não termos infecção e fazermos a desinfecção dele, até o frasco, limpando bem os equipos [...] (E1)

\section{Continuidade dos cuidados}

[...] é continuar dessa forma, cuidando bem do cateter, para não termos infecção e fazermos a desinfecção dele, até o frasco, limpando bem os equipos [...] (E1)

Cinco entrevistados optaram por manter a aplicação das práticas do pacote de medidas para prevenção de infecções associadas ao CVC sem novas sugestões, considerando que a prática já surtiu efeito e deve ser continuada.

\section{Uso de Equipamentos de Proteção Individual}

[...] o que eu já vi é que tem algumas enfermeiras que não utilizam a máscara para fazer o curativo[...] (E8)

Os Equipamentos de Proteção Individual (EPI) preconizados aos profissionais envolvidos no momento da inserção do CVC incluem gorro, máscara cirúrgica, óculos de proteção, avental estéril, luvas estéreis e campos estéreis, entretanto, a Agência Nacional de Vigilância Sanitária (ANVISA) não discorre sobre a obrigatoriedade da máscara cirúrgica na manutenção ou realização do curativo desse dispositivo (BRASIL, 2012).

Em relação à manutenção dos curativos dos cateteres, sugeriu-se que os auxiliares e técnicos de enfermagem também pudessem realizá-la:

[...] às vezes, a enfermeira não pode fazer o curativo. Está soltando, e você não vai pregar um micropore por cima. Eu não tenho coragem de fazer isso e já troquei muitas vezes o curativo à noite $[\ldots](E 5)$

Devido ao acúmulo de tarefas gerenciais e privativas, à medida em que observa o enfermeiro atarefado em demasia, auxiliares e técnicos de enfermagem acabam realizando a confecção do curativo do CVC, contudo, o Conselho Regional de Enfermagem de São Paulo (COREN) determina que os curativos devem ser realizados exclusivamente pelo Enfermeiro habilitado e capacitado, promovendo assim a inspeção, a palpação e a avaliação contínua do sítio de inserção do cateter (COREN-SP, 2014).

Além da equipe de enfermagem reconhecer a relevância de sua participação na implantação de um pacote de medidas, as práticas recomendadas devem ser significativas para os profissionais, de forma, a subsidiar o engajamento e comprometimento no manejo adequado e seguro na manipulação e manutenção do CVC em crianças.

\section{CONCLUSÃO}

Além de tratar-se de uma temática atual sobretudo na UTIP, a participação da equipe de enfermagem na implantação do pacote de medidas pode ser considerada de suma importância na crescente qualidade da assistência prestada às crianças, uma vez que tal intervenção visa reduzir o número de infecções relacionadas e associadas ao CVC e prevenir complicações clínicas durante a hospitalização infantil. Assim, cabe às instituições de saúde, a responsabilidade de promover educação permanente e continuada com o 
intuito de favorecer a manutenção das práticas científicas contidas no pacote de medidas em pediatria pela equipe de enfermagem.

\section{REFERÊNCIAS}

1. BRACHINE JDP, PETERLINI, MAS, PEDREIRA MLG. Método Bundle na redução de infecção de corrente sanguínea relacionada a cateteres centrais: revisão integrativa. Rev Gaúcha Enferm, 2012; 33(4): 200-10.

2. BRASIL. Agência Nacional de Vigilância Sanitária. Medidas de Prevenção de Infecção Relacionada à Assistência à Saúde. Brasília: 2th Anvisa, 2017.

3. BRASIL. Conselho Nacional de Saúde. Resolução no 466, de 12 de dezembro de 2012. Brasília: 2012.

4. COREN-SP. Conselho Regional de Enfermagem de São Paulo. Parecer CT COREN-SP 043 /2013. São Paulo: 2014.

5. DALLÉ J, KUPLICH NM, SANTOS RP, et al. Central venous catheter-related infection after the implementation of a preventive bundle in a intensive care unit. Rev HCPA. 2012; 32(1):10-7.

6. FORTUNATTI CFP. Impact of two bundles on central catheter-related bloodstream infection in critically ill patients. Rev. Latino-Am. Enfermagem [Internet]. 2017, 25:e2951.

7. GOMES, AVO; NASCIMENTO, MAL. Central venous catheterization in pediatric and neonatal intensive care units. Rev Esc enferm USP. 2013 Aug; 47(4): 794-800.

8. IHI. Institute for Healthcare Improvement. Improvement Stories: What is a Bundle? c2011.

9. LACERDA RA, NUNES BK, BATISTA AO et al. Evidence-based practices published in Brazil: identification and analysis of their types and methodological approaches. Rev Esc Enferm USP. 2011 Jun; 45(3):777-86.

10. MINAYO MCS, DESLANDES SF, GOMES R. Pesquisa Social: Teoria, método e criatividade. 25th ed: Vozes; 2012.

11. NOBRE ASP, MARTINS, MDS. Prevalência de flebite da venopunção periférica: fatores associados. Rev. Enf. Ref. 2018 Mar; serIV (16): 127-138.

12. OLIVEIRA, CR, NEVE ET, RODRIGUES EC, et al. Peripherally inserted central catheter in pediatrics and neonatology: Possibilities of systematization in a teaching hospital. Esc Anna Nery. 2014 July/Sept;18(3):379-85.

13. OLIVEIRA FT, STIPP MAC, SILVA LD, FREDERICO M, DUARTE SCM. Comportamento da equipe multiprofissional frente ao bundle do cateter venoso central na terapia intensiva. Esc. Anna Nery 2016; 20(1): 55-62.

14. ROSADO V, ROMANELLI RMC, CAMARGOS PAM. Fatores de risco e medidas preventivas das infecções associadas a cateteres venosos centrais. J Pediatr. 2011;87(6): 469-77.

15. SILVA SGD, NASCIMENTO ERPD, SALLES R KD. Bundle to prevent ventilator-associated pneumonia: a collective construction. Texto contexto - enferm. 2012 Oct/Dec; 21(4): 837-44.

16. SQUARÇA PB. Pacote de medidas para a prevenção de infecções causadas pela presença de cateter venoso central na criança. Dissertação (Mestrado em Enfermagem) - Centro de Ciências da Saúde. Universidade Estadual de Londrina, Londrina, 2015; 93 p. 